\title{
LAS PRÁCTICAS PROFESIONALES Y LAS PASANTÍAS DESDE LA LEGISLACIÓN COMPARADA*
}

\author{
THE PROFESSIONAL PRACTICES AND THE INTERNSHIPS \\ FROM THE COMPARED LEGISLATION \\ LES PRATIQUES PROFESSIONNELLES ET LES STAGES \\ DEPUIS LA LÉGISLATION COMPARÉE
}

\section{Alma Elena RUEDA RODRÍGUEZ**}

RESUMEN: El presente artículo estudia las prácticas profesionales y las pasantías como mecanismo de la transición escuela-trabajo desde una perspectiva comparada. El propósito es evidenciar, en primer lugar, la problemática que se presenta para la unificación conceptual; en segundo lugar se analiza la legislación de diversos países; en tercer lugar se señalan los trabajos realizados por la Organización Internacional del Trabajo y la Unión Europea, para concluir con la situación que afrontan algunos países de América Latina respecto al tema que nos ocupa.

Palabras clave: Prácticas profesionales, pasantías, transición escuela-trabajo.

ABSTRACT: This article studies internships and traineeships as a mechanism for the transition from school to work in a comparative perspective to demonstrate, in first place, the problems presented to unify concepts; secondly, legislation of different countries are analyzed to continue the study from the perspective of the International Labour Organization and the position of the European Union in these practices and the Youth Guarantee, to conclude the situation facing most of the Latin American countries regarding this issue.

* Recibido el 16 de marzo de 2014 y aceptado para su publicación el 27 de marzo de 2014.

Agradezco la lectura y observaciones realizadas al presente trabajo a Enrique Cruz, Pamela Padilla, Paulina Galicia y a Noemí Monroy.

** Estudiante de la Escuela Internacional de Doctorado en Formación de la Persona y Mercado del Trabajo ADAPT-CQIA de la Universidad de Bérgamo (Italia). Colaboradora de la Associazione per gli Studi Internazionali e Comparati sul Diritto del Lavoro e le Relazioni Industriali (a.elenarueda@gmail.com). 
Key Words: Internship, traineeship, transition school to work.

RÉSUMÉ: Le présent article étudie les pratiques professionnelles et les stages comme mécanisme de la transition école-travail depuis une perspective comparée. Le propos est de mettre en évidence en premier lieux la problématique qui se présente, pour l'unification conceptuelle, en second lieux, l'analyse de la législation de divers pays; en troisième lieux sont signalés les travaux réalisés par l'Organisation International du Travail et l'Union Européenne, pour conclure avec la situation qu'affrontent quelques pays d'Amérique latine en rapport avec le sujet qui nous concerne.

Mots-clés: Pratiques professionnelles, transition école-travail.

SUMARIO: I. Introducción. II. Una perspectiva comparada de las prácticas profesionales y las pasantías. III. Las prácticas profesionales bajo la perspectiva de la Organización Internacional del Trabajo y la Comisión Europea. IV. A manera de conclusión. V. Bibliografía.

\section{INTRODUCCIÓN}

a Organización Internacional del Trabajo (OIT) refiere que los jóvenes representan la promesa de un cambio positivo en las sociedades, específicamente si son vistos desde una perspectiva de la formación del capital humano, ya que son los principales actores dentro de las sociedades, y de ellos depende el futuro del mundo, toda vez que responderán a los desafíos próximos. No sólo nos referimos a los desafíos en materia laboral, sino también a aquellos de carácter económico, tecnológico, social y político; es por esto que los jóvenes son los actores dinamizadores de una sociedad, y de ahí se deriva la importancia del estudio de este sector poblacional.

En específico, el Banco Mundial establece la necesidad de invertir en los jóvenes para impactar de manera adecuada en su desarrollo a través de cinco fases: el estudio, el inicio de la etapa laboral, la adopción de un estilo de vida saludable, la formación de una familia y el ejercicio de sus derechos cívicos. ${ }^{1}$ Estas cinco fases abarcan distintas dimensiones en el desarrollo de los jóve-

1 Banco Mundial, El desarrollo y la próxima generación. Informe sobre el desarrollo mundial, 2007, disponible en: http:// www.oij.org/file_upload/publicationsItems/document/doc1202813603.pdf. 
nes, que a futuro afectarán al desarrollo de una vida satisfactoria, por lo que es ineludible que éstas se complementen en todos los aspectos para que a partir de su interacción puedan ayudar a que los jóvenes afronten los problemas de la actualidad. Pero de estas cinco fases, la educación y el inicio de la etapa laboral de los jóvenes son las etapas relevantes para el presente estudio.

En 2013, se calcula que 74.5 millones de jóvenes de entre 15 y 24 años de edad han estado desempleados, casi un millón más que en el año anterior. La tasa mundial de desempleo juvenil ha aumentado hasta el 13.1 por ciento, ${ }^{2} \mathrm{y}$ es precisamente este grupo de población el que tiene una marcada dificultad para ingresar al mercado de trabajo; la tasa de desempleo es hasta tres veces mayor que la de los adultos, y mientras que uno de cada tres adultos se encuentra en la informalidad, este porcentaje aumenta hasta la mitad en el caso de los jóvenes. Esta realidad hace que afronten una situación difícil y precaria que influye directamente en la calidad de vida y que tiene trascendencia a corto, mediano y a largo plazo. Específicamente en el aspecto laboral, si un joven comienza su vida profesional en un empleo informal, le será difícil encontrar un empleo formal en un futuro, fenómeno ya analizado bajo la noción de los scarring effects, ${ }^{3}$ que se presenta tanto frente al desempleo juvenil como cuando se está bajo una inestabilidad laboral o cuando el joven se encuentra justo en la transición escuela-trabajo, y lamentablemente, lo único que consigue es un empleo informal.

Debido a lo anterior, los mecanismos de transición escuela-trabajo juegan un papel fundamental para el futuro de los jóvenes. En el presente artículo nos dedicaremos específicamente al estudio de dos de ellos: las prácticas profesionales y las pasantías, ${ }^{4}$ que constituyen actualmente los mecanismos más utilizados en todo el mundo — pero también los más polémicos-.

Estos mecanismos sirven como un "puente" para acercar a los jóvenes al mercado de trabajo que permiten que el joven pueda poner en práctica los conocimientos teóricos adquiridos durante su formación profesional en un mundo "real", es decir, en situaciones concretas y existentes. Pero, ¿en realidad se sabe qué es una pasantía o una práctica profesional?, ¿cuáles son las

2 Organización Internacional del Trabajo, Tendencias mundiales del empleo 2014, enero de 2014.

3 Nordtröm Skans, Oskar, Scarring Effects of the First Labour Market Experience: A Sibling Bases Analysis, 2004.

4 En inglés denominados: internship y traineeship. 
diferencias entre ellas?, ¿cuál es el tipo de legislación que debe regularlas? En este tipo de prácticas, ¿`se establece una relación laboral? Y, finalmente, uno de los cuestionamientos más relevantes es si existe un marco internacional que regule estas prácticas en la región de América Latina.

Los conceptos de prácticas profesionales y pasantías son muy polémicos y presentan confusiones conceptuales porque se usan indistintamente; pero para efectos de este estudio se propondrá una definición para cada uno. Lo anterior sin la pretensión de establecer una definición única, ya que esta tarea es casi imposible, en virtud de que ambos términos varían dependiendo del país y del contenido de la legislación que los regula. A grandes rasgos, y para poder establecer una idea clara de estos mecanismos, podemos señalar que las prácticas profesionales generalmente forman parte de las actividades académicas, curriculares o de formación realizadas durante la universidad, siendo éstas la mayoría de las veces exigidas para poder obtener el grado académico. Generalmente es un crédito de carácter obligatorio durante el periodo educativo, ya que — como la propia palabra, "práctica", lo refiere—, el alumno está ejercitándose o poniendo en ejecución algún conocimiento obtenido durante sus estudios. Es entonces que el "practicante" es también un estudiante que, durante sus últimos años de universidad, realiza un periodo en una empresa, ya sea privada o pública, con el fin de ejercitarse para un futuro laboral. Cabe destacar que este periodo no establece ninguna relación laboral entre el practicante y la empresa, toda vez que se realiza durante el periodo escolar como complemento a la educación.

Por otro lado, las pasantías son definidas por el Diccionario de la Real Academia Española ${ }^{5}$ como el ejercicio del pasante en las facultades y profesiones; entendiéndose como pasante a la persona que asiste y acompaña al maestro de una facultad en el ejercicio de ella, para imponerse enteramente en su práctica.

Se debe subrayar que las pasantías pueden formar o no parte de los estudios técnicos o profesionales. En algunas ocasiones éstas se realizan cuando un joven ya ha obtenido el título o grado académico y tienen como objetivo el que esta experiencia constituya un "puente" que lo aproxime al mercado de trabajo, y se genere así la posibilidad de que las empresas en las que se

\footnotetext{
5 Diccionario de la Real Academia Española, 22a. ed., 2001, disponible en: bttp:/ / lema.rae. es/drae/.
} 
desempeñen dichas prácticas puedan establecer un contrato laboral, siendo éste en primera instancia de tiempo determinado por su propia razón de ser, existiendo la posibilidad de que al final del periodo de pasantías el joven se integre a la empresa con un contrato de tiempo indefinido.

Es importante referir que las definiciones dadas anteriormente no pueden ser consideradas como únicas, porque como ya se ha dicho con antelación, ambas definiciones — prácticas profesionales y pasantías - obedecen a las características dadas por las legislaciones de cada país, como se estudiará a continuación.

\section{UNA PERSPECTIVA COMPARADA DE LAS PRÁCTICAS PROFESIONALES Y LAS PASANTÍAS}

\section{Argentina}

En Argentina este mecanismo de transición escuela-trabajo se realiza a través de las prácticas profesionalizantes y las pasantías educativas.

Las pasantías educativas se encuentran reguladas por la Ley $26.427,{ }^{6}$ que las considera como el conjunto de actividades formativas que realizan los estudiantes en empresas y organismos públicos o empresas privadas con personería jurídica, sustantivamente relacionado con la propuesta curricular de los estudios cursados en unidades educativas, que se reconocen como experiencia de alto valor pedagógico, sin carácter obligatorio. Se debe mencionar que éstas tienen un régimen de excepción, por lo cual no constituyen una relación laboral y son tipificadas como una relación especial.

La duración mínima es de 2 meses y hasta un máximo de 12 meses, aunque existe la posibilidad de renovarlas por 6 meses más. Periodo donde el joven tiene un horario de no más de 6 horas al día o de 12 horas semanales, con la posibilidad del pago de una retribución o la asignación de algún estímulo, en ambos casos fijo y determinado por el convenio colectivo de la actividad correspondiente, pero que nunca podrá constituir un salario.

Los jóvenes pueden realizar estas pasantías en empresas privadas o en organismos públicos y deben tener una edad mínima de 18 años.

\footnotetext{
6 Publicada el 26 de noviembre de 2008.
} 
Los principales objetivos de este tipo de contratos son:

- Servir al proceso de orientación profesional, mejorando las posibilidades de inserción laboral.

- Constituir un nexo entre la producción y la educación.

- Complementar la formación académica.

- Incorporar saberes, habilidades y actitudes vinculados a situaciones reales.

- Aumentar el conocimiento y manejo de tecnologías vigentes.

Las prácticas profesionalizantes están reguladas en la Ley de Educación Técnico-Profesional núm. 26.058, en la que se establece que son aquellas estrategias formativas integradas en la propuesta curricular, y tienen como propósito consolidar, integrar y ampliar las capacidades según el perfil profesional correspondiente; son organizadas por la institución educativa, siendo un requisito para la aprobación de carreras técnicas a nivel medio superior y, específicamente, de educación técnico-profesional. En ningún caso los alumnos serán sustituidos por trabajadores.

\section{Chile}

En Chile, las pasantías y prácticas profesionales no se encuentran reguladas por ninguna legislación federal; sin embargo, se presume que este tipo de prácticas se lleva a cabo por los jóvenes, ya que el Código de Trabajo menciona que tales prácticas no darán origen a un contrato de trabajo.

Por otro lado, las prácticas profesionales y pasantías se encuentran limitadas sólo a los estudiantes de carreras universitarias en el área de la salud. La definición de "prácticas profesionales" la encontramos en el Dictamen ordinario núm. 5428/258, emitido por la Dirección de Trabajo, ${ }^{8}$ donde se refiere que una práctica profesional debe entenderse en un sentido amplio

7 Dictamen ordinario núm. 1983/100. No dan origen a un contrato de trabajo los servicios prestados por un alumno o egresado de una institución de educación superior o de la enseñanza media técnico-profesional, durante un tiempo determinado, para cumplir con el requisito de práctica profesional.

8 La Dirección del Trabajo emite dictámenes en su función de fijar el sentido y alcances de las leyes de trabajo. 
como el ejercicio de cualquier arte o facultad conforme a sus reglas, realizado por cierto tiempo para que los estudiantes se habiliten y puedan ejercitar los actos propios de su profesión. De este modo, se comprenden en ella todas las actividades de este tipo, efectuadas durante el transcurso de los estudios y no sólo las que se verifican al final de los mismos; en calidad de egresado, estas prácticas son las relativas a las que se llevan a cabo dentro del sistema educativo. Debe destacarse que cada universidad regula el funcionamiento de las prácticas profesionales, ya que no existe una legislación federal específica ${ }^{10}$ que se encargue de ello. El Dictamen también define a las pasantías como las estadías de perfeccionamiento de posgrado en el extranjero de los médicos cirujanos, farmacéuticos, químico-farmacéuticos, bioquímicos, cirujano-dentistas y otros profesionales, ${ }^{11}$ que constituyen actividades de perfeccionamiento de los estudios universitarios posteriores al título de grado, cuyo título o diploma da derecho a percibir la asignación de perfeccionamiento de estudios de especialización. ${ }^{12}$

En ausencia de una legislación federal o local específica en la materia toda vez que las prácticas profesionales y las pasantías son sólo reguladas por estos dos decretos-, ambas figuras se practican sin tener un marco normativo que establezca formalmente qué son, cuál es su duración, cuáles son sus objetivos, así como cuáles son los derechos y obligaciones entre las partes.

Lo anterior evidencia que en Chile no existe un marco legislativo específico sobre estos mecanismos de transición escuela-trabajo.

9 Cfr. bttp:/ / wmw.dt.gob.cl/ legislacion/1611/w3-article-89001.html.

10 Por ejemplo, la Universidad de Chile tiene un Reglamento de los Estudios Universitarios de Pregrado (Decreto Universitario Exento núm. 0017946, del 7 de agosto de 2008), en el que se establece que las prácticas profesionales son parte de la formación de pregrado.

11 Profesionales referidos en los incisos $a$ y $b$ del artículo 5o. de la Ley 19.378.

12 Dictamen ordinario núm. 5428/258: 1) Las pasantías consistentes en estadías de perfeccionamiento de posgrado en el extranjero, de los profesionales referidos en las letras a) y b) del artículo 5o. de la Ley 19.378, constituyen actividades de perfeccionamiento de posgrado cuyo título o diploma da derecho a percibir la asignación de perfeccionamiento de posgrado que contempla el inciso final del artículo 42 de la ley 19.378. 2) El pago de la asignación de perfeccionamiento de posgrado es, sin perjuicio del derecho del funcionario para solicitar el reconocimiento del respectivo curso o estadía de posgrado, como actividad de capacitación para los efectos de la carrera funcionaria. 3) Las entidades administradoras de salud primaria municipal pueden ejercer el nombramiento provisional de director de establecimiento de atención primaria de salud municipal, por alguna de las modalidades de contratación que permite el artículo 14 de la Ley 19.378, porque dicho nombramiento provisional no puede ejercerse en calidad de interino o de subrogante. 


\section{Colombia}

En Colombia las prácticas profesionales no se encuentran reguladas en una ley en específico; sin embargo, el Decreto núm. 933 de 2003, en su artículo 7o., ${ }^{13}$ establece que las prácticas y/o programas no constituyen contratos de aprendizaje, ${ }^{14}$ y el Proyecto de Ley 185 de $2011^{15}$ hace referencia a éstas, estableciendo que las pasantías o prácticas empresariales son un proceso sistemático desarrollado por un estudiante de educación superior en instituciones públicas o privadas, organizaciones no gubernamentales u organizaciones comunitarias, donde se ponen en práctica los conocimientos adquiridos durante su carrera educativa para realizar actividades que generen un impacto específico, es decir, son un requisito académico para poder graduarse de la carrera profesional o técnica. ${ }^{16}$

Las universidades o entidades educativas están facultadas para regular este tipo de prácticas, ${ }^{17}$ por lo que generalmente realizan un convenio entre ellas y el estudiante (practicante estudiantil).

\section{España}

España es uno de los países que regula específicamente este tipo de prácticas para jóvenes, las cuales son: prácticas no laborales, prácticas curriculares y extracurriculares.

La primera regulación sobre las prácticas de los estudiantes universitarios, en específico de los programas de cooperación educativa, fue por primera vez abordada por el Real Decreto 1497/1981, ${ }^{18}$ ahora derogado, que tenía como

13 Es decir, las prácticas profesionales no son un contrato de aprendizaje ni tampoco son consideradas un trabajo, ya que no se encuentran reguladas por el código sustantivo del trabajo.

14 Diferenciando a éste como un contrato regulado por el derecho laboral, sin subordinación y por un plazo no mayor de dos años.

15 Cfr. http://servoaspr.imprenta.gov.co:7778/gacetap/gaceta.mostrar_documento?p_tipo=05 óp_nu mero $=185$ dop_consec $=28369$.

16 Por ejemplo en las licenciaturas de derecho, medicina, odontología, comunicación social, etcétera.

17 Por ejemplo, el Instituto de Educación Empresarial tiene un Reglamento sobre Prácticas y Pasantías que puede verse en: http:// wmw.idee.edu.co/site/documentos/Reglamentos_p_p.pdf.

18 Decreto del 19 de junio de 1981 (derogado). Cfr. http://wmw.boe.es/buscar/doc.php?id= BOE-A-1981-16532. 
objeto conseguir una formación integral del estudiante universitario a través de programas de cooperación educativa con las empresas para la formación de los alumnos de los dos últimos cursos de la facultad, escuela técnica superior o escuela universitaria, y tenía como características comunes que estos programas no establecían relación contractual alguna sobre el estudiante y la empresa, ya que la relación era estrictamente académica y no laboral.

Con posterioridad, el Real Decreto 1497/198719 estableció directrices generales comunes de los planes de estudio de los títulos universitarios de carácter oficial y validez en todo el territorio nacional, y dividió las enseñanzas universitarias en una estructura cíclica, incorporando al sistema el cómputo del haber académico por créditos. Éste fue modificado por el Real Decreto 1947/1981,20 el cual dispuso que en las universidades se podrían establecer programas de cooperación educativa con las empresas para la formación de los alumnos que, aún cursando los créditos universitarios, hubieran superado el 50 por ciento de los créditos necesarios para obtener el título universitario.

El Real Decreto 1707/201121 regulaba las prácticas académicas externas de los estudiantes universitarios, las cuales constituían una actividad de naturaleza formativa realizada por los estudiantes universitarios y supervisada por las universidades, cuyo objetivo era permitir a los mismos aplicar y complementar los conocimientos adquiridos en su formación académica, favoreciendo la adquisición de competencias que les preparen para el ejercicio de actividades profesionales, que les faciliten su empleabilidad y que fomenten su capacidad de emprendimiento, pudiendo realizarse en la propia universidad o en entes colaboradores, tales como empresas, instituciones y entidades públicas y privadas, en el ámbito nacional e internacional. Estas prácticas en ningún caso implican una relación laboral entre la empresa y el estudiante.

El Real Decreto 1543/2011,22 vigente en la actualidad, regula las prácticas no laborales dirigidas a jóvenes en empresas o grupos empresariales que for-

19 Decreto del 27 de noviembre de 1987 (derogado), en bttps:// wmw.boe.es/diario_boe/txt. php? $i d=B O E-A-1987-27707$.

20 Real Decreto del 19 de junio de 1981 (derogado), en: http://mmm.boe.es/buscar/doc. php? id=BOE-A-1981-16532.

21 Real Decreto del de 18 de noviembre de 2011, en: https://mmm.boe.es/diario_boe/txt. php? id=BOE- A-2011-19362.

22 Real Decreto del 2 de octubre de 2011, http:// wmm.boe.es/boe/dias/2011/11/18/pdfs/ BOE-A-2011-18062.pdf. 
malicen convenios con los servicios públicos de empleo, entendiéndose que éstas, en ningún caso, supondrán una relación laboral.

Las principales características de las aludidas prácticas son:

- Son dirigidas a jóvenes de entre 18 y 25 años.

- Los jóvenes pueden tener o no una titulación oficial universitaria, titulación de formación profesional, de grado medio o superior, o titulación del mismo nivel que el de esta última, correspondiente a las enseñanzas de formación profesional, artísticas o deportivas, o bien un certificado de profesionalidad.

- Las prácticas se desarrollarán en centros de trabajo de la empresa o del grupo empresarial.

- Están bajo la dirección y supervisión de un tutor.

- Tendrán una duración de entre tres y nueve meses, y al final de éstas se expedirá un certificado.

\section{Italia}

En Italia, el mecanismo de transición escuela-trabajo recibe el nombre de prácticas ${ }^{23}$ y representa una medida de la política pública que tiene como fin crear contacto directo entre el sujeto hospedante (en este caso la empresa privada o pública) y el practicante, con el fin de enriquecerlo de conocimiento, de competencias profesionales y de integración al trabajo. Lo anterior, a través de un periodo de orientación laboral o de formación en una situación en la que no se configura una relación de trabajo.

Los diferentes tipos de prácticas reconocidas son:

- Prácticas obligatorias (tirocini curriculari): promovidas por las universidades, las escuelas y los centros de formación profesional. Son todas las experiencias que tienen lugar dentro de un curso formal de estudios o de formación.

- Prácticas formativas y de orientación o prácticas laborales (tirocini formativi e di orientamento): están diseñadas para facilitar la elección de carrera,

23 Para efectos de facilitar la lectura a las lectoras y lectores hispanohablantes se ha preferido traducir este mecanismo como "práctica", si bien se ha dejado en aras de una mayor claridad conceptual el término italiano tirocinio. 
con la finalidad de contribuir a mejorar la empleabilidad de los jóvenes y ofrecerles un primer contacto con la realidad laboral. Los destinatarios son personas que han obtenido un título dentro de los 12 meses anteriores. Su duración no puede ser superior a seis meses.

- Prácticas de inserción al mercado laboral o para la reinserción (tirocini di inserimento/reinserimento): estas prácticas también están dirigidas a las personas con discapacidad. Su duración no puede ser superior a doce meses.

La regulación de estas prácticas se encuentra en la Linee-guida in materia di tirocini del 24 de enero de 2013,24 y es un cuadro de referencia común a todas las regiones y provincias autónomas de Italia, ${ }^{25}$ por lo que ésta establece cuáles son los estándares mínimos, cuál es su duración, cuáles son las partes y cuáles sus obligaciones, así como la forma para proceder para la activación de estas prácticas, estableciendo que el sujeto promotor debe implantar un tutor para cada practicante, el cual garantizará que los objetivos sean cumplidos, existiendo un monitoreo de las prácticas por cada provincia con el fin de realizar reportes nacionales.

Italia, sin duda, es uno de los países que posee una óptima regulación sobre el mecanismo.

\section{México}

En México, las prácticas profesionales se desarrollan solamente dentro del ámbito escolar, precisamente a nivel universitario. De esta manera, los jóvenes que están estudiando en instituciones de educación superior se encuentran con un requisito obligatorio para obtener el título universitario o grado académico: el presentar un "servicio social", ${ }^{26}$ mientras que las pasantías son

24 Linee-guida in materia di tirocini del 24 gennaio 2013. Cfr. http:/ / wmw.funzionepubblica. gov.it/media/1061502/accordo_tirocini_24012013.pdf.

25 Para mayor información sobre las prácticas en Italia puede verse la ponencia "Tirocini: una mappatura completa”, presentada en la Conferencia Internacional Internship and Traineeship for Students and Young People, Training, School-to-Work Transition or Exploitation? (Bérgamo, 25-26 de octubre de 2013) por U. Buratti y F. Fazio, disponible en: http://prezi.com/ jpkemxwldnwmc/ tirocini-una-mappatura-completal.

26 Como ya se explicó anteriormente, el servicio social en México es una especie de práctica profesional, por lo que se le nombrará de forma indistinta entre estos dos términos. Aun- 
realizadas por los jóvenes que finalizaron sus estudios pero que aún no obtienen la certificación correspondiente para la obtención del título o grado en comento. A pesar de que en el país existe una normativa que regula de forma general qué son las pasantías, ésta no establece claramente cómo es que se deben regular tales prácticas, que son desempeñadas por los jóvenes para poder facilitar su entrada al mercado laboral.

El servicio social se encuentra regulado por la Ley Reglamentaria del Artículo 5o. constitucional, relativo al ejercicio de las profesiones en el Distrito Federal, concretamente en el capítulo VII, "Del servicio social de estudiantes y profesionistas", ${ }^{27}$ así como en el Reglamento para la Prestación del Servicio Social de los Estudiantes de las Instituciones de Educación Superior en la República Mexicana. En ninguna de estas dos normativas se encuentra una definición precisa respecto a qué es el servicio social o las prácticas profesionales. ${ }^{28} \mathrm{El}$ servicio social tiene como objetivo el de contribuir a la formación académica y capacitación profesional del estudiante; es importante mencionar que esta práctica se debe realiza cuando el joven se encuentra estudiando y tiene el 70 por ciento de los créditos académicos previstos en el programa de estudios correspondiente o cuando éste se haya finalizado, por lo que la universidad en la que se encuentre estudiando el joven será la encargada de garantizar que se cumpla con el objetivo de la práctica profesional. Las mencionadas prácticas no pueden emplearse para cubrir necesidades de tipo laboral o institucional, es decir, no tendrán la categoría de trabajador. Las prácticas o servicio social tienen como duración seis meses como mínimo y dos años como máximo, pudiendo tener una remuneración si el servicio social absorbe totalmente las actividades del estudiante o del profesionista.

que originalmente el servicio social tenía como finalidad que los estudiantes universitarios contribuyeran a la sociedad con los conocimientos que adquirieron durante su preparación. De hecho, el servicio social nace en la Facultad de Medicina de la UNAM, con la finalidad de apoyar a sectores desprotegidos de atención médica.

27 Artículos 52 a 62 de la Ley de las Profesiones en el Distrito Federal.

28 Sin embrago, se puede considerar la definición que la Universidad Nacional Autónoma de México, siendo la casa máxima de estudios universitarios en el país, regula el servicio social universitario en el Reglamento General del Servicio Social. El artículo 3o. establece que se entiende por servicio social universitario la realización obligatoria de actividades temporales que ejecuten los estudiantes de carreras técnicas y profesionales, tendentes a la aplicación de los conocimientos que hayan obtenido y que impliquen el ejercicio de la práctica profesional en beneficio o en interés de la sociedad. 
Respecto a las pasantías, el artículo 30 de la Ley Reglamentaria del Artículo 5o. constitucional establece que la Dirección General de Profesiones podrá extender autorización a los pasantes de las diversas profesiones para ejercer la práctica respectiva por un término no mayor de tres años; sin embargo, esta ley no establece una definición clara sobre qué son estas prácticas formativas; es decir, la ley no precisa claramente una definición de pasantías, así como no regula adecuadamente si los pasantes deberán tener un plano formativo, un tutor o una remuneración.

\section{Perú}

Perú es de los pocos países de América Latina que tiene un marco normativo específico sobre el tema de las pasantías y las prácticas profesionales, ya que cuenta con una Ley sobre las Modalidades Formativas Laborales, ${ }^{29}$ así como con un Reglamento.

La ley describe que las prácticas profesionales y las pasantías relacionan el aprendizaje teórico y práctico mediante el desempeño de tareas programadas de capacitación y formación profesional.

Las prácticas profesionales ${ }^{30}$ de capacitación laboral juvenil se caracterizan por realizar el proceso formativo en las unidades productivas de las empresas, permitiendo a los beneficiarios ejercitar su desempeño en una situación real de trabajo, teniendo como finalidad que los jóvenes de entre 16 y 23 años que no hayan culminado o hayan interrumpido la educación básica, o que habiéndola culminado no siguieran sus estudios de nivel superior, sean técnicos o universitarios, adquieran los conocimientos teóricos y prácticos en el trabajo, a fin de incorporarlos a la actividad económica en una ocupación específica. Se debe realizar un convenio de capacitación laboral juvenil, en el cual la empresa se obligue a brindar facilidades a la persona para que realice su aprendizaje práctico durante el tiempo que dure el convenio, mediante la ejecución de tareas productivas correspondientes a un programa en específico de capacitación, que será previamente definido por la empresa anualmente.

La empresa tiene la responsabilidad de planificar y diseñar los programas, así como de dirigir, administrar, evaluar y certificar las actividades formati-

\footnotetext{
29 Ley núm. 28518 sobre las Modalidades Formativas Laborales.

30 Las prácticas profesionales en Perú son aquellas prácticas que se realizan cuando el joven está fuera del sistema educativo.
} 
vas. Los jóvenes se obligan a cumplir las tareas productivas de la empresa conforme a la reglamentación y normatividad de ésta. La ley establece un número máximo de beneficiarios en capacitación laboral juvenil, es decir, estos jóvenes no pueden exceder del 20 por ciento del total del personal del área u ocupación específica, ni del 20 por ciento del total de trabajadores de la empresa con vinculación laboral directa. Este límite puede incrementarse en un 10 por ciento, siempre y cuando este último porcentaje esté compuesto exclusivamente por jóvenes con discapacidad, así como por jóvenes madres con responsabilidades familiares.

En el caso de empresas que cuenten con más de tres y menos de diez trabajadores, sólo se podrá suscribir convenio con un joven beneficiario bajo esta modalidad. El tiempo en que deban de realizarse las prácticas no será mayor a seis meses en ocupaciones de poca calificación, o de poca o ninguna complejidad, así como en niveles menores de responsabilidad en el ejercicio de la ocupación, pudiendo ser prorrogable hasta por un periodo similar y no mayor a veinticuatro meses en ocupaciones que requieren mayor calificación y, por ende, mayor complejidad con mayores niveles de responsabilidad en el ejercicio de su ocupación. En la misma empresa, los periodos de capacitación laboral intermitentes o prorrogados no pueden exceder en su conjunto de doce meses o veinticuatro meses, según corresponda. Al final de esta capacitación se podrá tener la posibilidad de obtener una certificación para que se reconozca una habilitación laboral técnica, la cual será reconocida por el Ministerio de Educación, siempre y cuando se haya cumplido con los requisitos solicitados.

En relación con las pasantías, se establece que son también una modalidad formativa que se realiza en las unidades productivas de las empresas y que buscan relacionar al beneficiario con el mundo del trabajo, en donde implementa, actualiza y contrasta lo aprendido en el centro de formación y se informa de las posibilidades de empleo existentes y de la dinámica de los procesos productivos de bienes y servicios. En esta modalidad de formación se ubica tanto a los beneficiarios como a los estudiantes de los últimos grados del nivel de educación secundaria de los centros educativos que necesiten, por razones formativas y curriculares, realizar una pasantía en la empresa y que tiene como finalidad reforzar la capacitación laboral adquirida e inicie, desarrolle o mejore las habilidades sociales y personales relacionadas con el ámbito laboral. La duración de este convenio no podrá ser mayor de tres meses. 
Respecto a la remuneración económica mensual, ${ }^{31}$ no puede ser inferior a una remuneración mínima cuando la persona en formación cumpla la jornada máxima prevista para cada modalidad formativa. Para el caso de jornadas formativas de duración inferior, el pago de la subvención es proporcional; con la excepción de las pasantías, cuya subvención aplicable a estudiantes de educación secundaria no podrá ser menor al cinco por ciento de la remuneración mínima; en los demás casos de pasantías, la subvención mensual no puede ser inferior al treinta por ciento de la remuneración mínima, y sólo en el caso de que la pasantía se realice asociada a las necesidades propias de un curso obligatorio requerido por el centro de formación profesional, el pasante no recibirá subvención alguna. Es importante destacar que la ley indica una subvención y nunca una remuneración.

\section{LAS PRÁCTICAS PROFESIONALES BAJO LA PERSPECTIVA DE LA ORGANIZACIÓN INTERNACIONAL DEL TRABAJO Y LA COMISIÓN EUROPEA}

El desempleo juvenil es también una de las situaciones más preocupantes para la Unión Europea, por lo que los mecanismos de transición escuelatrabajo son de los temas más importantes. Por lo cual, en 2010 el Parlamento Europeo adoptó un informe de iniciativas sobre el fomento del acceso de los jóvenes al mercado laboral, reforzando el estatuto del becario, del periodo de prácticas y del aprendiz, ${ }^{32}$ en el que pide a la Comisión elaborar una Carta Europea de Calidad de las Prácticas que facilite estadísticas sobre los periodos de dichas prácticas y elabore un estudio comparativo sobre los diferentes regímenes de ésta existentes en los Estados miembros.

En 2012, la Comisión Europea presentó el estudio de una visión global de los acuerdos de periodos de prácticas en los Estados miembros; los resultados de la consulta indican que en muchos países la dimensión educativa de los

31 La remuneración económica se encuentra contemplada en el artículo 47 de la Ley núm. 28518.

32 Parlamento Europeo, Informe sobre el fomento del acceso de los jóvenes al mercado de trabajo y refuerzo del estatuto del becario, del periodo de prácticas y del aprendiz, 14 de junio de 2010, disponible en: bttp:/ / www.europarl.europa.eu/sides/getDoc.do?pubRef=-//EP//NONSGML+REPORT+A7-20 10-0197+0+DOC+PDF+VO//ES. 
periodos de prácticas ha disminuido, ya que se pide a los becarios que lleven a cabo tareas que no contribuyen al progreso de su desarrollo profesional, por lo que dicha Comisión hizo especial énfasis en la necesidad de crear un marco de calidad para los periodos de prácticas. El objetivo de la consulta fue recabar opiniones sobre cómo mejorar la calidad de los periodos de prácticas a través de la creación de este marco, que tiene como fin ayudar a los jóvenes titulares de carrera a la transición de la educación al trabajo. A lo largo de este año, veintidós Estados miembros recibieron recomendaciones específicas para cada país, destinadas a mejorar las diversas situaciones de los jóvenes en el mercado laboral, y se presentó la recomendación de la Comisión relativa a los principios para la alta calidad en las prácticas, la cual busca, entre otros objetivos, apoyar a los mecanismos que cuenten con un contenido de aprendizaje cualitativo y que tengan paralelamente una transparencia y calidad de la información de las prácticas profesionales y las pasantías. Adicionalmente a lo anterior, con el fin de evitar el riesgo de esta generación de jóvenes, y con el fin de afrontar de manera sistemática la falta de empleo, la Unión Europea ha puesto en marcha la iniciativa denominada "garantía juvenil", ${ }^{33}$ la cual tiene como objetivo principal hacer que todos los jóvenes menores de 25 años reciban una oferta cualitativamente válida de trabajo mientras continúan con sus estudios; esta oferta de trabajo deberá efectuarse dentro de los cuatro meses del inicio del desempleo o la salida del sistema de educación formal.

La garantía juvenil es una de las reformas estructurales más importantes y urgentes que los Estados miembros de la Unión Europea deben introducir para abordar el desempleo juvenil y la inactividad y, adicionalmente, para mejorar la transición escuela-trabajo. En resumen, la lógica de la garantía juvenil es muy simple, ya que pretende asegurarse de que ningún joven se quede en paro o inactivo durante más de cuatro meses.

La garantía juvenil debería permitir a los jóvenes encontrar un trabajo adecuado a su formación o que les permita adquirir una educación, habilidades y experiencia que son directamente relevantes para aumentar sus posibilidades de encontrar un trabajo en el futuro. Fue entre 1980 y 1990 cuando los países nórdicos de Europa ${ }^{34}$ implementaron la garantía juvenil para su población

\footnotetext{
33 Mejor conocida en inglés como youth guarantee.

34 Países como Suecia, Noruega, Dinamarca y Finlandia, y recientemente Austria, Alemania, Países Bajos y Polonia.
} 
joven, y son justo estos países quienes se encuentran más comprometidos, ya que diecisiete Estados miembros ${ }^{35}$ de la Comisión Europea presentaron el 15 de enero de 2014 sus planes para aplicar la garantía juvenil para ayudar a todas las personas menores de 25 años y desempleadas a encontrar un empleo, a contar con educación continua y un aprendizaje o un periodo de prácticas dentro de los cuatro meses siguientes de su situación de desempleo o que hayan dejado la educación formal.

Desde otro punto de vista, la Organización Internacional del Trabajo, respecto al tema de las pasantías, refiere que con frecuencia son consideradas una excelente manera para adquirir experiencia laboral y afianzarse en el mercado de trabajo, pero que, paralelamente, son un riesgo latente para los jóvenes, ya que se han presentado numerosas denuncias de abusos, pues éstas son consideradas como fuente de trabajo a bajo costo o, incluso, gratuito. En específico sobre el tema de la garantía juvenil, en un comunicado de prensa ${ }^{36}$ reconoció que se encuentra lista para aunar esfuerzos con la Comisión Europea a fin de apoyar a los Estados miembros en la implementación de programas para los jóvenes, reconociendo que esta garantía para la juventud puede ser muy eficaz para reducir el desempleo y el desaliento en el que se encuentra, destacando que paralelamente se necesita supervisión y evaluación de su impacto.

De lo anterior se puede concluir que a nivel internacional —refiriéndonos, en específico, a la Organización Internacional del Trabajo y a la Comisión Europea - se están implementando mecanismos orientados a la mejora de la situación laboral de los jóvenes y que existe la preocupación latente por el desempleo, las malas prácticas de las pasantías y las prácticas profesionales. Lo lamentable es que esta preocupación sólo se ve reflejada en los países pertenecientes a la Unión Europea, pues son éstos quienes están actuando directamente a través de recomendaciones específicas, informes, estudios comparados, consultas, planes y marcos de referencia. El gran cuestionamiento sobre lo anterior es ¿qué se está haciendo en América Latina para establecer una garantía juvenil?, ¿quién establece un marco regulatorio con derechos y obligaciones mínimos para los pasantes y practicantes para los países de

35 Los Estados comprometidos son: Bélgica, Bulgaria, Croacia, Chipre, República Checa, Francia, Alemania, Grecia, Hungría, Irlanda, Italia, Letonia, Lituania, Polonia, Portugal, Rumania, Eslovaquia, Eslovenia, España, Suecia y Reino Unido.

36 Noticia del 4 de abril de 2013, en: http:// wmw.ilo.org/global/ about-the-ilo/newsroom/ news/ WCMS_209302/lang--es/index.htm. 
América Latina?, ¿quién supervisa estas prácticas en la región? Las pasantías y prácticas profesionales ya se encuentran reguladas por una legislación nacional en la mayoría de los países de esta región, sin embargo, no existe una guía o un marco de referencia dirigido a los países de América Latina que contenga las características mínimas que requieren estos mecanismos y que paralelamente incluya la famosa garantía juvenil; garantía que se encuentra íntimamente ligada con la transición escuela-trabajo. Es entonces que la problemática que se afronta en América Latina es difícil y complicada, ya que si bien estos mecanismos de transición escuela-trabajo se practican en la mayoría de los países estudiados, también lo es el hecho de que no existe una "guía" que oriente a todos los países hacia la adopción de un patrón similar. En otras palabras, es deseable que se contemplen características mínimas para estas prácticas, para que condichos estándares mínimos se pueda comenzar a pensar en la forma de activar la garantía juvenil en esta región.

\section{A MANERA DE CONCLUSIÓN}

A partir del estudio realizado entre distintos países de América Latina, España e Italia, podemos evidenciar que este mecanismo de transición escuela-trabajo es utilizado de manera constante pero, contemporáneamente, se presentan diversas problemáticas sobre el tema... La primera, como ya se ha mencionado, radica en la definición, ya que las prácticas profesionales reciben diversos nombres; por ejemplo, en Argentina, Chile, Italia y Perú son llamadas prácticas profesionales o profesionalizantes, mientras que en Colombia se les denomina prácticas estudiantiles; en México servicio social, y en España prácticas no laborales. Lo anterior refleja que sobre el tema no se encuentra establecido un concepto de práctica profesional general, ya que esta definición dependerá siempre de las características dadas por la legislación que las regule, dependiendo de la nación que se estudie; es decir, en un país podrá llamarse de una forma u otra, pero por sus características sabemos que encuadra dentro de nuestras definiciones establecidas al principio de este artículo (prácticas profesionales o pasantías).

Desde la misma lógica se desprende que si bien estas prácticas se encuentran contempladas por algún tipo de legislación en cada uno de los países estudiados, la mayoría de las veces el ordenamiento que las normaliza no se 
encuentra legislado de manera adecuada, ya que están reguladas por decretos o por ámbitos diversos, generalmente relacionados con la educación y la formación. Lo anterior torna más complicada la situación en la que se encuentran las prácticas profesionales y las pasantías, porque si bien este tipo de mecanismo ofrece una serie de ventajas para los jóvenes y empleadores, también lo es que existe el peligro latente de que las referidas prácticas, al no contar con una regulación adecuada y específica en la que se establezca claramente qué son, cuáles son sus objetivos, cuáles son los derechos y obligaciones de los jóvenes practicantes o pasantes, cuál es su duración, quién la supervisará y quién certificará el conocimiento, nos afrontamos entonces ante una terrible situación que conlleva a que las fuertes críticas del mecanismo de transición escuela-trabajo tengan razón de ser, ya que, en realidad, lejos de ser un trampolín para acceder al mercado laboral, son herramientas utilizadas de forma "perversa" por los empleadores, y es entonces que los jóvenes terminan estancándose en las actividades que realizan, sin conseguir conocimientos nuevos que los preparen para un futuro laboral exitoso y, consecuentemente, corren el riesgo de que terminen realizando tareas que fueron contempladas para los trabajadores, es decir, sin ningún contenido educativo, y por lo tanto concluyan siendo mano de obra gratuita.

Sumándose a la problemática anterior, se debe destacar que el problema específico de América Latina es aún más grave, si tomamos en cuenta que esta región no tiene un marco de referencia para poder regular apropiadamente estas prácticas, por lo que cada país regula el mecanismo libremente, sin ninguna guía o pauta que ayude a establecer características comunes en la región. Es por esto que existen diferentes denominaciones y diferentes características, evidenciando esta problemática el objetivo de la investigación que nos ocupa, misma que es enfatizar la necesidad latente para que las pasantías y prácticas profesionales sean reguladas de forma general.

De esta manera, existirán pautas mínimas del tema y, consecuentemente, serán utilizadas adecuadamente; si éstas tuvieran una "guía", nos referimos específicamente a un marco de referencia o a una recomendación, como la que establece la Unión Europea, se podría instaurar un contenido específico que refleje la calidad en estas prácticas y pasantías y, mejor aún, se podría crear un plan específico para implementar la garantía juvenil en América Latina, que es la clave al problema del desempleo juvenil. Es entonces que nos cuestionamos: ¿será la OIT la mejor vía para establecer un marco de referencia de 
estos mecanismos de transición escuela-trabajo?, ¿será ella quien pueda orientar a América Latina sobre la implementación de la garantía juvenil en esta región? A nuestro punto de vista, la Organización Internacional de Trabajo ayudaría mucho a mejorar la situación de la región en estos dos temas claves para el futuro de los jóvenes.

\section{BIBLIOGRAFÍA}

ABDALA, E., La inclusión laboral de los jóvenes: entre la desesperanza y la construcción colectiva, Montevideo, CINTEFOR-OIT, 2005.

ARIAS GILBERT, E. N., "Algunas consideraciones sobre discriminación, igualdad de trato y represalia".

BAlLESTER PASTOR, María Amparo, El contrato de formación y otras formas de contratación laboral en alternancia, Valencia, Tirant Lo Blanch, 2000.

BANCO Mundial, El desarrollo y la próxima generación. Informe sobre el desarrollo mundial, 2007.

CINTERFOR-OIT, Empleo y capacitación laboral de jóvenes en América Latina, disponible en: http:// wmw.oei.es/etp/empleo_capacitacion_laboral_jovenes_AL.pdf.

CockX, Bart, "Scarring Effects of Remaining Unemployed for Long-Term Unemployed School-Leavers", disponible en: http://politiquessociales.net/ $I M G / p d f / d p 5937 . p d f$.

Córdova, Efrén, "Del empleo total al trabajo atípico: chacia un viraje en la evolución de las relaciones laborales?”, Revista Internacional del Trabajo, Ginebra, vol. 1105, núm. 4, octubre-diciembre de 1986.

GALlart, María Antonia, La formación para el trabajo y los jóvenes en América Latina, 2003.

GNECCO, Lorenzo P., Contratos de formación y derecho del trabajo; aprendizaje, becas, pasantías y otras prácticas formativas. Modelos de actuación práctica, Buenos Aires, Astrea-Depalma, 2002.

GonZÁLEZ-VelosA, Carolina, ¿Cómo mejorar las oportunidades de inserción laboral de los jóvenes en América Latina?, Banco Interamericano de Desarrollo, 2012, disponible en: http:// idbdocs.iadb.org/wsdocs/getdocument.aspx?doc num = 36976183. 
GrisoliA, J. A., Derechos del trabajo y de la seguridad social, 13a. ed., Buenos Aires, Abeledo-Perrot, 2008, t. I.

— vista de Derecho Laboral y Seguridad Social.

IBARrola, María, Escuela, capacitación y aprendizaje, disponible en: http:/ / wmw. oitcinterfor.org/sites/default/files/file_publicacion/ibarro2.pdf.

JARA MALES, Patricia, Redes de protección social. Mejores respuestas para adolescentes y jóvenes, Banco Interamericano de Desarrollo, 2013, disponible en: bttp:/ / www.oitcinterfor.org/sites/default/files/file_publicacion/11915.pdf.

MolTó GARcíA, Juan Ignacio, La nueva contratación laboral, Madrid, Montecorvo, 1999.

NORDTRÖM SKANS, Oskar, Scarring Effects of the First Labour Market Experience: A Sibling Bases Analysis, 2004, disponible en: bttp://www.ifau.se/ upload/ pdf/se/2004/wp04-14.pdf.

ORGANIZACIÓN IBEROAMERICANA DE LA JUVENTUD, "La juventud en Iberoamérica. Tendencias y urgencias", 2007, disponible en: bttp://www.oij. org/file_upload/publicationsItems/document/doc1202813603.pdf.

OrganizACión INTERnACIONAL DEL TraBAJO, "El déficit de trabajo decente un nuevo informe de la OIT esboza las nuevas tendencias globales del empleo", Revista Trabajo, núm. 56, abril de 2006.

- L La crisis del empleo juvenil: un llamado a la acción, Ginebra, 2012.

, "Trabajo decente y juventud", 2013, disponible en: http://www.odisea.org.mx/Biblioteca/Jovenes/Trabajo\%20decente $\% 20 y \% 20 j u v e n t u d \% 20$ en $\% 20$ AL\%202010.pdf.

ORGANIZACIÓN PARA LA COOPERACIÓN Y DESARROLLO ECONÓMICOS, ¿Dónde se sitúa México?, 2012.

PARLAMENTO EUROPEO, Informe sobre el fomento del acceso de los jóvenes al mercado de trabajo y refuerzo del estatuto del becario, del periodo de prácticas y del aprendiz, 14 de junio de 2010, disponible en: http://www.europarl.europa. eu/sides/getDoc.do?pubRef=-//EP//NONSGML+REPORT+A7-2010$0197+0+D O C+P D F+V 0 / / E S$.

y OIT, "Políticas para la acción", 2013, disponible en: http://www. ilo.org/wcmsp5/groups/public/---americas/---ro-lima/documents/presentation/ woms_223119.pdf. 
Programa PARA LA COHESIÓn SOCIAL DE AmÉRICA LATINA, Estado del arte sobre empleo juvenil en América Latina y Europa, disponible en: http:/ / www.oei. es/70cd/Estado ArtEmpleJuvenil.pdf.

RAMA Vitale, Claudio, "La incorporación de la práctica estudiantil en las universidades latinoamericanas como nuevo paradigma educativo", Universidades, vol. LXI, núm. 48, enero-marzo de 2011, disponible en: bttp:/ / www. redalyc.org/pdf/373/37319199006.pdf.

RODRÍGUEZ, Laura, "Políticas públicas para promover el empleo juvenil y el emprendedurismo de los jóvenes en México", disponible en: http:/ / prejal. oit.org.pe/prejal/docs/emp_juvenil_y_emprendedurismo_mexico.pdf.

VERA, Alejandro y CASTIONI, Remi, "Los jóvenes en Latinoamérica. La transición escuela-trabajo como objeto de las políticas públicas", Boletín Técnico SENAC, Río de Janeiro, vol. 36, núm. 2, mayo-agosto de 2010, disponible en: bttp:/ / www.senac.br/BTS/362/artigo1.pdf. W/ 